International Journal of Engineering \& Technology, $7(3.20)(2018) 475-479$
International Journal of Engineering \& Technology
SPC
Website: www.sciencepubco.com/index.php/IJET
Research paper

\title{
GFRecog: a Generic Framework with Significant Feature Selection Approach for Face Recognition
}

\author{
${ }^{1}$ Riyadh Rahef Nuiaa , ${ }^{2}$ Seif Ali Abdulhussein and ${ }^{3}$ Bahaa Kareem Mohammed \\ ${ }^{I}$ Education college, Wasit University \\ ${ }^{2}$ college of computer science and mathematics, Kufa University \\ ${ }^{3}$ kut technical institute, middle technical university \\ *Corresponding Author Email: riyadh@uowasit.edu.iq
}

\begin{abstract}
Identification of Humans uniquely is given paramount importance in the contemporary world. It is evident in applications of all fields so as to ensure secure and accurate transactions. Out of many approaches biometric approach became a dependable mechanism for this purpose. Face is one of the biometrics that plays vital role in recognizing humans across the globe. Many approaches came into existence for face recognition. In this paper we proposed a generic framework known as GFRecog that is extendable to support future methods of face recognition as well. We propose a methodology for face recognition using Gabor wavelets by extracting significant features from training dataset and perform matching operation with the given input image. Projection of face images onto a feature space that reflects diversity of face images is considered an efficient approach. Our approach works with faces that are captured under different lighting conditions, expression and pose. We built a prototype application using MATLAB with a benchmark dataset to demonstrate the proof of concept. The empirical results revealed that the accuracy of the proposed face recognition method is significantly high.
\end{abstract}

Keywords: Face, significant feature set, face recognition, similarity, generic framework

\section{Introduction}

Face recognition is one of the important features that are widely used in different applications. The rationale behind this is that face is one of the biometrics that provides unique identity to people across the globe. There are many applications that are based on face recognition. It can be used in applications like Human Computer Interaction (HCI), telecommunications, security and surveillance. The faces of humans vary from person to person. Therefore, it is important to use it for recognizing humans. There are many ways in which features of an image are extracted. The features are used to find similarity and recognize faces. There are many ways in which features can be extracted and face can be recognized. There are many challenges in face recognition. They are related to varying lighting conditions, pose conditions and expression conditions. There are local extraction methods and global extraction methods. There are many methods. For instance, holistic image based methods, local feature extraction methods and hybrid methods that combine both local feature extraction and holistic approaches.

Principal component analysis is one of the methods used. Generally face recognition is done with two phases. They are known as training phase and testing phase. In the training phase, a classification model is built in order to have knowledge that can be used in order to recognise faces. When pose, illumination and expression are different it is not easy to have accurate face recognition. In this paper, we proposed a method that takes care of face recognition under varying expression conditions, pose conditions and lighting conditions. Gabor wavelets are used in this paper in order to recognize faces. The remainder of the paper is structured as follows. The remainder of the paper is structured as follows. Section II reviews literature on face recognition techniques and their applications. Section III presents the proposed face recognition approach. Section IV presents experimental results. Section V concludes the paper besides providing recommendations for future work.

\section{Related Works}

This section provides review of works related to face recognition. [1] proposed two techniques for face recognition. They are known as Wash-Hadamard Transform (WHT) and facial feature extraction. They found that their proposed algorithm was to provide very close results with respect to face recognition. [2] proposed a face recognition system based on neural networks and image processing. They used DCT and Self-Organizing Map (SOM) for achieving this. They made experiments in MATLAB in order to evaluate their face recognition system. [3] proposed a method for face recognition. They named it as face recognition based on Sparse Representation based classification (SRC). It is the concept which is in the generalized form of nearest feature subspace and nearest neighbour. They tested it using Yale B and AR datasets collected from UCI machine learning repository. [4] focussed on Human Computer Interaction (HCI). They explored gesture recognition with different phases such as recognition, tracking, and detection.

[5] proposed a face recognition approach that is based on finding local geometrical signatures. Their method was found computationally efficient face recognition. They also used Support Vector Machine (SVM) as part of face recognition system. They achieved very high verification rates. [6] a condition based algorithm for face recognition. It is known as Locally-Constrained Collaborative Representation (LCCR). This approach works in 
collaborative fashion with local features taken in constrained way. It is able to improve robustness and discrimination in recognition. [7] followed different approach for face recognition. They used similarity metric learning for the purpose. It was an unconstrained face verification based on learning metrics for similarity. [8] focused on automatic face recognition system. They employed face recognition for another technique known as automatic facial makeup detection. They also used adaptive pre-processing scheme for improving accuracy of face recognition.

[9] explored various face recognition techniques available. They covered both datasets available and the methodologies used on the datasets for face recognition. They found many challenges in face recognition due to the invariants and various light conditions. In the same fashion, [10] made a review of different applications where face recognition techniques are used. They focused on both common methods of face recognition and applications that are useful in the real world. There was another survey on face recognition from [11]. They explored different face recognition methods and compared them in terms of memory usage, speed, computational overhead and accuracy in face recognition. [12] proposed a novel alignment based approach for face recognition. They used different datasets available in the real world for evaluating their work. [13] used two techniques for face recognition. They are known as PSO-based feature selection and transform domain feature extraction. These two techniques are combined in order to have better results in the accuracy of recognition.

[14] also combined two techniques for performing face recognition. They are known as discrete wavelet transform and Zernike moments. Their algorithm is known as near infrared face recognition algorithm. They achieve less computational cost when compared with online face recognition systems. [15] focused on the purpose of domain-specific development of face memory. The reason behind this is that the memory based approach is used to have face perception.

[16] proposed a face recognition system which is pose invariant They employed techniques like Principal Component Analysis
(PCA) and ANFIS. Their system was working with high recognition ratio with neuro-fuzzy analysis. [17] focused on singe image based face recognition. They used filters like sobel and Gabor besides local ternary patterns. They used algorithms like local ternary pattern (LTP) and Principal Component Analysis (PCA). When compared with original LBP their approach was performing better. [18] focused on enhancing face recognition using PCA and Discrete Wavelet Transform (DWT). DWT is used to extract

facial features and then recognition is done with the help of features.

[19] proposed a robust face recognition method that is based on multi-subregion based correlation filter bank (MS-CFB). This method exploits the benefits of local-based and global-based feature extraction methods for robust face recognition. [20] focused on the concept of general-purpose face recognition. They proposed a framework known as OpenFace which is used to recognize faces and used as part of mobile applications. These applications made use of library of faces in order to evaluate face recognition. In this paper we proposed a new face recognition approach that is capable of recognition of faces with different pose, light and expression conditions. The proposed technique is based on Gabor wavelet.

\section{Proposed Methodology for Face Recognition}

We proposed a methodology used for face recognition. The database contains both training and testing datasets. Gabor filter is used to have analysis on the part of face recognition. The feature extraction is used to extract features on the given input image and the features of training dataset are compared for similarity. The rationale behind this is that the features and their similarity can help in accurately recognizing faces. The comparison module is responsible to have similarity and make a decision. Recognize face is the process of recognizing face.

\section{Database}

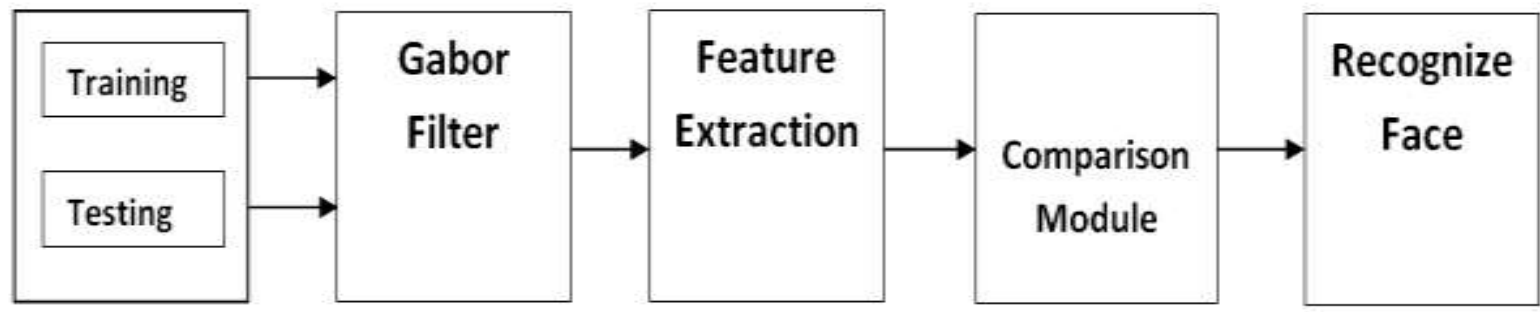

Figure 1: Methodology for face recognition

Gabor wavelets are widely used for obtaining features of images that can be used in order to find useful results. For instance, it can be used to recognize faces. In other words, it is used for pattern recognition. Wavelets on the other hand are also known for distortion tolerant features. Wavelets are found to be very appropriate in order to find facial frequencies. Gabor wavelets also have features that are identical. The notion of Gabor feature bank is used to extract features from given face image. Gabor filter is able to get native features from face image. Moreover, a twodimensional Gabor filter is considered as Gaussian function which is shown in Eq. (1).

$\psi_{\mu, v}(\mathrm{Z})=\frac{\left\|K_{\mu, v}\right\|^{2}}{\sigma^{2}} e^{\frac{\left\|K_{\mu, v}\right\|^{2}\|z\| \|^{2}}{2 \sigma^{2}}}\left[e^{i k_{\mu, v} Z}-e^{\frac{\sigma^{2}}{2}}\right]$

where $\mu, v$ is orientation and scale of Gabor kernels. $\mathrm{Z}=(\mathrm{x}, \mathrm{y}),\|$. denotes norm factor. Then the wave vector is computed as in Eq. (2).
$K_{\mu, v}=K_{v e^{i \phi_{u}}}$

$K_{v}=K_{-} \max / f^{v}$ and $\emptyset_{\mu}=\pi \mu / 8$ represents the spacing factor between kernels in frequency domain. The Gabor kernel is computed as shown in Eq. (3).

$O_{\mu, v}(\mathrm{Z})=\mathrm{A}(\mathrm{Z}) * \psi_{\mu, v}(\mathrm{Z})$

$\mathrm{Z}=(\mathrm{x}, \mathrm{y})$ and $*$ operator denotes convolution operator.

\section{Datasets Used}

Two datasets are used for making experiments. The datasets are collected in such a way that they are captured in various lighting conditions, pose and expression conditions. 


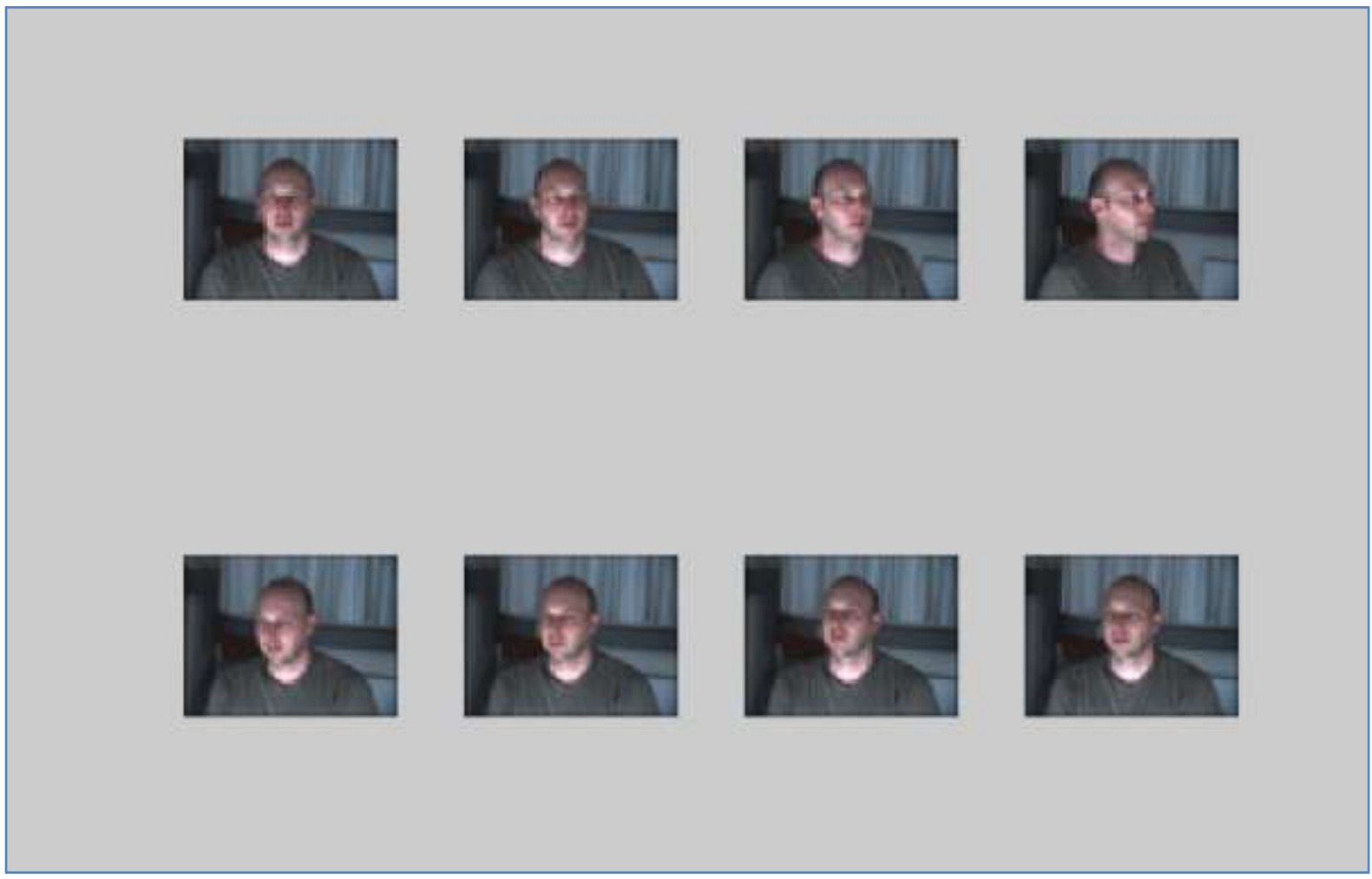

Figure 2: Dataset 1

This is the dataset containing a human with different expressions, poses and the images are captured in different lighting conditions.

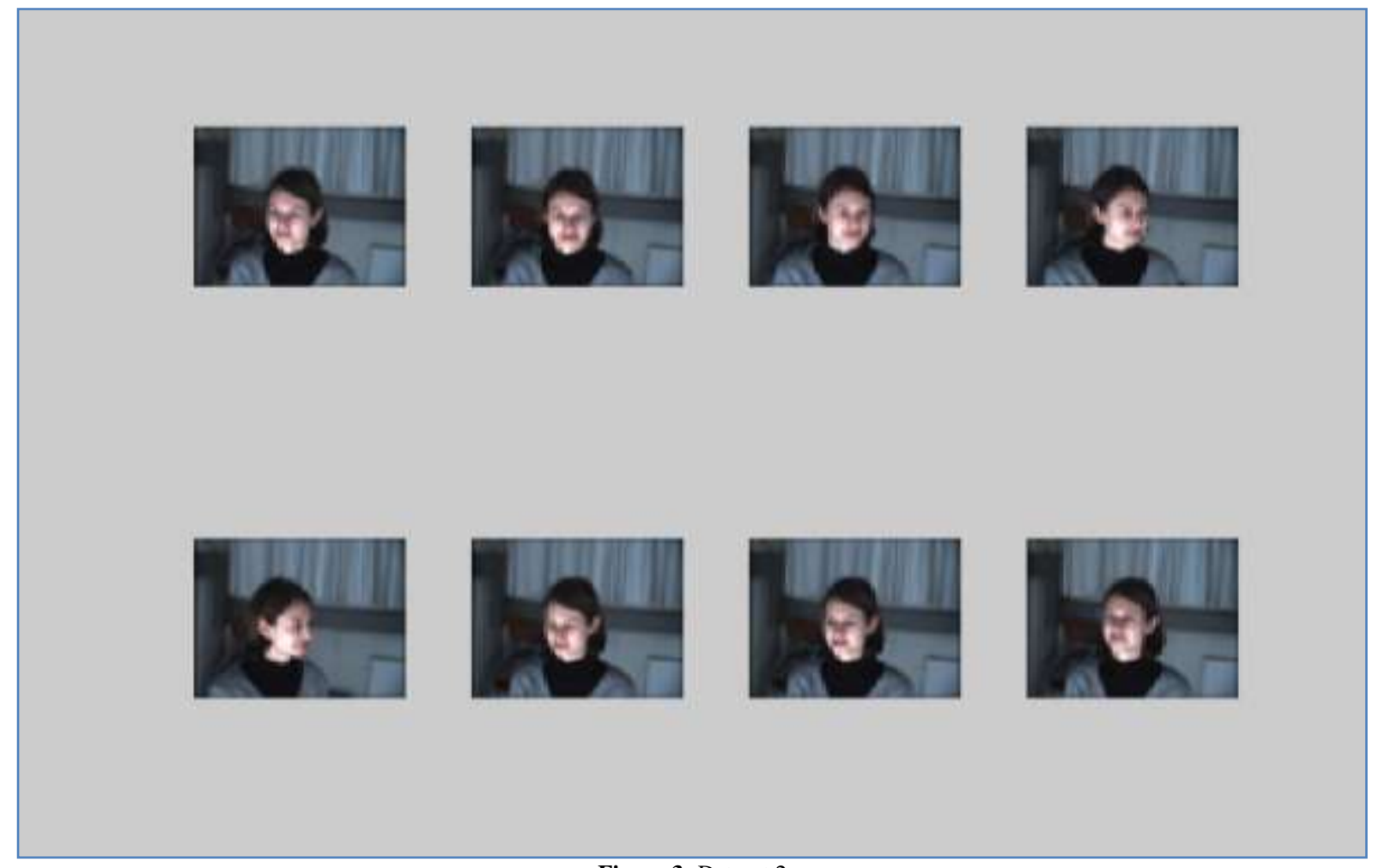

Figure 3: Dataset 2

This is the dataset containing a human with different expressions, poses and the images are captured in different lighting conditions. These two datasets are used for empirical study. There are training and testing phases in the proposed methodology. In the training phase a model is built for knowledge while testing phase is meant for actual face recognition.

\section{Faces Recognized Results}

The results of face recognition revealed that the proposed system is working accurately. The results of four sets of experiments are presented in Figure 4, Figure 5, Figure 6 and Figure 7. 

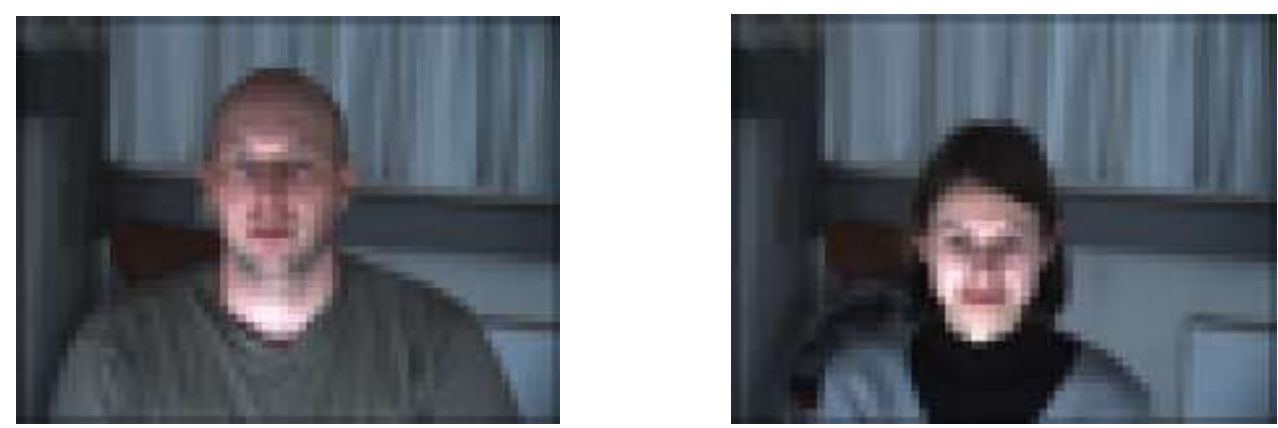

Figure 4: True positive results of experiment 1
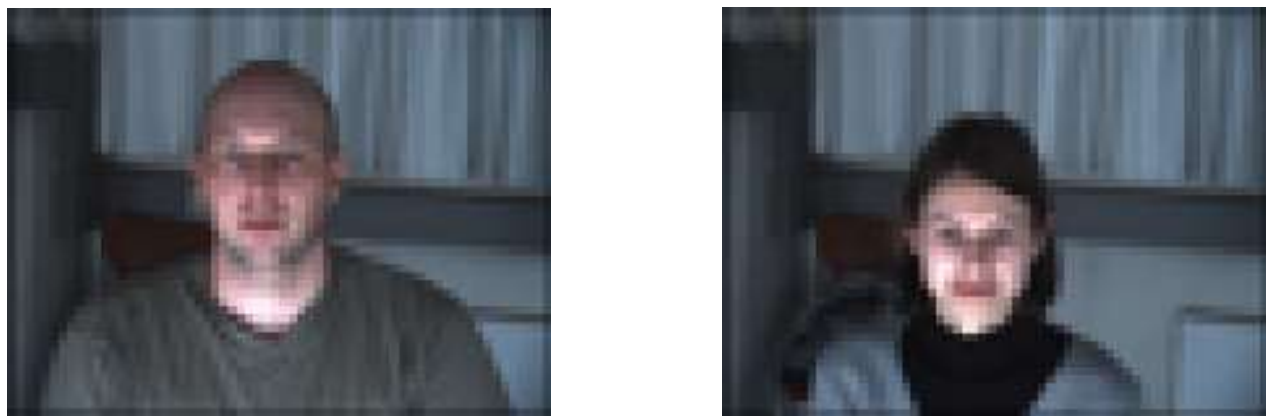

Figure 5: True positive results of experiment 2
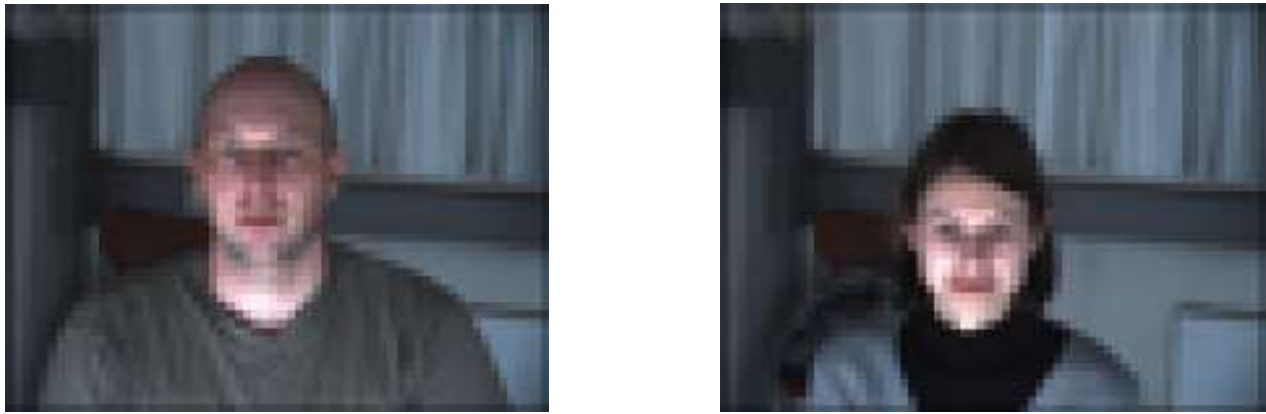

Figure 6: True positive results of experiment 3
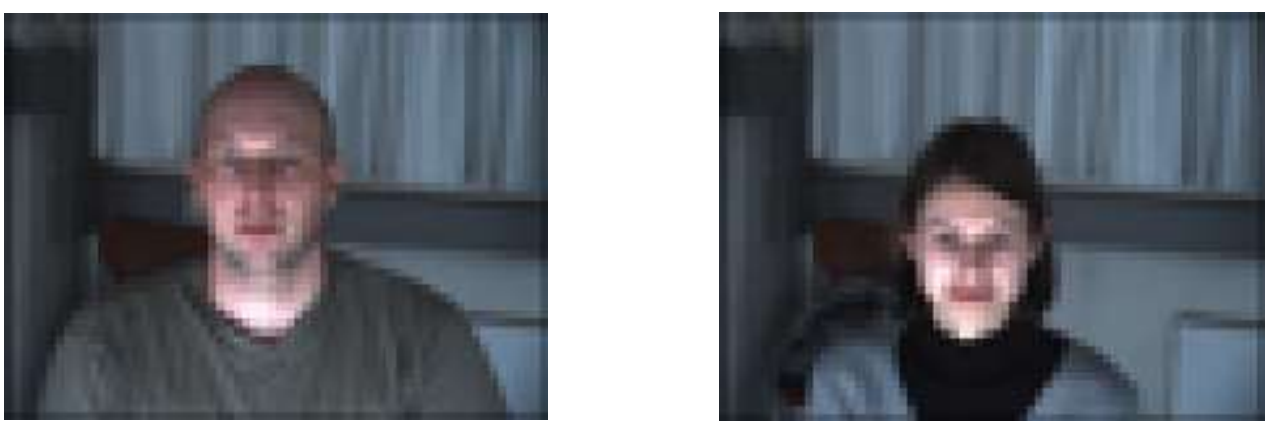

Figure 7: True positive results of experiment 4

The result of four experiments revealed that the face recognition is done successfully with different pose and expression conditions.

\section{Performance Evaluation}

The experiments are also done with YCRBR, HSV, GRAY and NTSC. The results of evaluation of the proposed approach are presented in Table 1.
Table 1: Shows classification results of different methods

\begin{tabular}{|c|c|c|c|c|}
\hline Method & YCRBR & HSV & GRAY & NTSC \\
\hline Classification Percentage & 68 & 65 & 50 & 85 \\
\hline
\end{tabular}

As sown in Table 1, it is evident that, the classification percentage is high with NTSC. The lease one is when GREY approach is used. The results are presented with respect to classification percentage. 
As presented in Figure 8, it is clear that the performance of classification different with different approaches. The face recognition in this paper is done with the proposed methodology that proved to be effective. The results revealed that it can be used in real time applications. The biometric face recognition is widely used in the real world which can be used to ensure that humans are accurately identified.

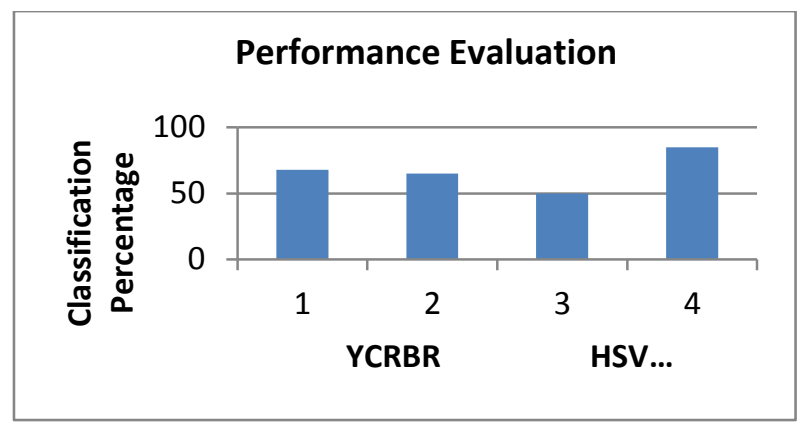

Figure 8: Shows classification performance with different method

\section{Conclusions and Future Work}

In this paper we studied various face recognition methods. We found in the literature that there are many face recognition approaches. However, face recognition under varying pose, light and expression condition is found to be an open problem to be addressed. In this paper we proposed a methodology for face recognition. Our methodology is based on Gabor filter and wavelet transform. In other words Gabor wavelets are used to have feature extraction and finding similarity between faces. The training dataset is used to have feature extraction. These features are then compared with the testing dataset in order to recognize face. We built a prototype application using MATLAB. The application is user-friendly and provides different options to have interactive verification of face recognition. It also throws light into different methods as presented in the evaluation of this paper. In future we focus on using a hybrid approach for more effective face recognition application.

\section{References}

[1] M. Hassan, I. Osman, and M. Yahia. (2007). Walsh-Hadamard Transform for Facial Feature Extraction in Face Recognition.PROCEEDINGS OF WORLD ACADEMY OF SCIENCE, ENGINEERING AND TECHNOLOGY. 23 , p194-198.

[2] Jawad Nagi, Syed Khaleel Ahmed and Farrukh Nagi. (2008). A MATLAB based Face Recognition System using Image Processing and Neural Networks. 4th International Colloquium on Signal Processing and its Applications. P83-88.

[3] Can-Yi Lu, Hai Min, Jie Gui , Lin Zhu and Ying-Ke Lei . (2012) Face recognition via Weighted Sparse Representation. Elsevier. P17.

[4] Siddharth S. Rautaray - and Anupam Agrawal. (2012). Vision based hand gesture recognition for human computer interaction: a survey, p1-54.

[5] Yinjie Lei, mohammedbennamoun, munawarhayat and yulanguo (2013). An efficient 3Dfacerecognitionapproachusinglocal geometricalsignatures. Patternrecognition. P1-16.

[6] Xi PENG, Lei ZHANG, ZHANG Yi and Kok Kiong Tan. (2013). Learning Locality-Constrained Collaborative Representation for Robust Face Recognition. P1-16.

[7] Qiong Cao, Yiming Ying and . (2013). Peng Li. IEEE. P2408-2415.

[8] Cunjian Chen,Antitza Dantcheva and Arun Ross. (2013). Automatic Facial Makeup Detection with Application in Face Recognition. 6th IAPR International Conference on Biometrics. P18.

[9] Reza Shoja Ghiass, Ognjen Arandjelovi, Abdelhakim Bendada and Xavier Maldagu. (2014). Infrared Face Recognition: A
Comprehensive Review of Methodologies and Databases. Elsevier. P1-57.

[10] Divyarajsinh N. Parmar and Brijesh B. Mehta. (2013). Face Recognition Methods \& Applications. IJCTA. 4 (1), p84-86.

[11] Jigar M. Pandya, Devang Rathod and Jigna J. Jadav. (2013). A Survey of Face Recognition approach. International Journal of Engineering. 3 (1), p632-635

[12] Xavier Fontaine, Radhakrishna Achanta and Sabine S"usstrunk. (2014). FACE RECOGNITION IN REAL-WORLD IMAGES. P15.

[13] N.L. Ajit Krisshna, V. Kadetotad Deepak, Manikantana and S. Ramachandran. (2014). Face recognition using transform domain feature extraction andpso-based feature selection. Applied Soft Computing. 22, p141-161.

[14] sajadfarokhi,sitimariyamshamsuddin,U.U.Sheikh,janflusser,moham madkhansari and kouroshjafari-Khouzani.(2014) Nearinfraredfacerecognitionbycombiningzernikemomentsandundec imateddiscretewavelettransform.digitalsignalprocessing. P1-15.

[15] Sarah Weigelt, Kami Koldewyn,Daniel D. Dilks, Benjamin Balas, Elinor mckone and Nancy Kanwisher. (2014). Domain-specific development of face memory but not face perception. 17 (1), p4758.

[16] Reecha Sharma and M.S. Patterh. (2015). A new pose invariant face recognition system using PCA and ANFIS. Optik. 126 , p34833487.

[17] Pronaya Prosun Das,Taskeed Jabid and S.M. Shariar Mahamud . (2015). Single Image Face Recognition based on Gabor, Sobel and Local Ternary Pattern. International Journal of Computer Applications. 132 (16), p1-19.

[18] Sikandar Afridi, Muhammad Irfan Khattak, Nasim Ullah, Gulzar Ahmad and Muhammad Shafi. (2015). Performance Enhancement Of Face Recognition System Using Principal Component Analysis Merged With Discrete Wavelet Transforms. ISSN. 34 (1), p76-83.

[19] Yan Yan, Hanzi Wang and David Suter. (2016). Multi-Subregion Based Correlation Filter Bank for Robust Face Recognition. Patten Recognition. P1-47.

[20] Brandon Amos, Bartosz Ludwiczuk and y Mahadev Satyanarayanan. (2016). Openface: A general-purpose face recognition library with mobile applications. P1-20. 